\title{
High Prevalence of Cannabidiol Use Within Male Professional Rugby Union and League Players: A Quest for Pain Relief and Enhanced Recovery
}

\author{
Andreas M. Kasper \\ Liverpool John Moores University \\ S. Andy Sparks \\ Edge Hill University \\ Matthew Hooks, Matthew Skeer, Benjamin Webb, Houman Nia, \\ James P. Morton, and Graeme L. Close \\ Liverpool John Moores University
}

\begin{abstract}
Rugby is characterized by frequent high-intensity collisions, resulting in muscle soreness. Players consequently seek strategies to reduce soreness and accelerate recovery, with an emerging method being cannabidiol (CBD), despite anti-doping risks. The prevalence and rationale for CBD use in rugby has not been explored; therefore, we recruited professional male players to complete a survey on CBD. Goodness of fit chi-square $\left(\chi^{2}\right)$ was used to assess CBD use between codes and player position. Effects of age on use were determined using $\chi^{2}$ tests of independence. Twenty-five teams provided 517 player responses. While the majority of players had never used CBD $(p<.001, V=0.24), 26 \%$ had either used it $(18 \%)$ or were still using it $(8 \%)$. Significantly more CBD use was observed in rugby union compared with rugby league $(p=.004, V=0.13$ ), but player position was not a factor $(p=.760, V=0.013)$. CBD use increased with players' age $(p<.001, V=0.28)$, with mean use reaching $41 \%$ in the players aged 28 years and older category $(p<.0001)$. The players using CBD primarily used the Internet $(73 \%)$ or another teammate $(61 \%)$ to obtain information, with only $16 \%$ consulting a nutritionist. The main reasons for CBD use were improving recovery/pain (80\%) and sleep (78\%), with $68 \%$ of players reporting a perceived benefit. These data highlight the need for immediate education on the risks of $\mathrm{CBD}$, as well as the need to explore the claims regarding pain and sleep.
\end{abstract}

Keywords: CBD, cannabis, DOMS, supplement

Rugby is a high-intensity collision sport that involves lowintensity aerobic activities (e.g., standing, walking, and jogging) combined with frequent periods of intermittent, intensive anaerobic activities (e.g., sprinting, side stepping, passing, and collisions; Austin \& Kelly, 2013; Twist et al., 2014). In 1895, the game of rugby was split into two variations-"league" and "union"-and, despite differences in laws, the two games share many common modalities with both based around frequent high-speed collisions. As a consequence of these repeated collisions, players from both codes of rugby experience increased markers of inflammation (Cunniffe et al., 2011) and muscle soreness (Twist et al., 2012). The muscle soreness in rugby is particularly pronounced, given it is a combination of both exercise- and impact-induced muscle damage (Naughton et al., 2017). This pain is often debilitating, affecting everyday function, and can last several days postgame and remain throughout the entire season (Fletcher et al., 2016). Players and support staff, therefore, constantly strive to improve recovery

Kasper, Hooks, Skeer, Webb, Nia, Morton, and Close are with the Research Institute for Sport and Exercise Sciences, Liverpool John Moores University, Liverpool, United Kingdom. Sparks is with the Sport Nutrition and Performance Research Group, Department of Sport and Physical Activity, Edge Hill University, Ormskirk, United Kingdom. Close (G.L.Close@ljmu.ac.uk) is corresponding author. strategies and reduce pain with an emerging method being the use of cannabidiol (CBD).

It is important to appreciate the fundamental difference between cannabis and CBD. The cannabis plant contains over 100 cannabinoids, with the two most well-known being CBD and tetrahydrocannabinol (THC). Although the exact nomenclature has been argued (Piomelli \& Russo, 2016), broadly speaking, the cannabis plant has two major strains-Cannabis indica and Cannabis sativa. The Cannabis sativa L-strain, more commonly referred to as hemp, must contain less than $0.2-0.3 \%$ dry weight THC, whereas other strains can contain up to $30 \%$ dry weight THC and are often referred to as marijuana. Many European countries have recognized the commercial value of hemp, and a legal limit of $0.2-0.3 \% \Delta^{9}$-THC is usually applied (Pellati et al., 2018). This difference in THC concentration is crucial, given that THC is the major psychotropic substance in cannabis, and it is often the primary reason why marijuana is grown, whereas hemp is grown for a variety of reasons, including clothing, shoes, rope, insulation, biofuel, protein supplementation, and to produce CBD as a nutritional supplement and/or medicine. While there are country to country variations in the legality of CBD, in the United Kingdom, CBD can be legally sold, providing that it does not contain more than $1 \mathrm{mg}$ of the controlled drug (or $1 \mu \mathrm{g}$, in the case 
of lysergide or any other $\mathrm{N}$-alkyl derivative of lysergamide) in the final "container," that is, in the bottle or packet that it is sold in (United Kingdom Government Home Office, 2020). Almost all other cannabinoids, except CBD, remain subject to the Misuse of Drugs Act 1971. There are a variety of forms of CBD that are commercially available for sale, including oils, capsules, sprays, tinctures, and gummies. From an athletic perspective, numerous claims have been made to suggest the possible benefits for athletic populations.

It has been reported that CBD possesses anxiolytic, antiinflammatory, anti-oxidative, and analgesic properties (Bergamaschi et al., 2011; Mechoulam \& Hanus, 2002), as well as suggested that it improves sleep quality (Russo et al., 2007). Importantly, it has also been suggested that CBD has a relative safe adverse effects profile (Bergamaschi et al., 2011), with side effects including tiredness and changes in appetite and weight (Iffland \& Grotenhermen, 2017). The majority of research into CBD and inflammation has been performed in rodent models (Xiong et al., 2012), and therefore the evidence supporting its practical application with athletes, at present, remains weak (Close et al., 2019). Moreover, CBD research is usually performed in combination with other cannabinoids, commonly THC (van de Donk et al., 2019), in something referred to as the entourage effect (Russo, 2019), raising doubts over the efficacy of pure CBD supplementation for athletes.

Although it is possible to produce synthetic CBD, in Europe, CBD must come from an industrial hemp strain than is European Union approved or come from outside the European Union. Given that CBD must come from the hemp plant, it is of no surprise that traces of THC are usually found in CBD products. While, legally, there must be less than $1 \mathrm{mg}$ of THC in the final container, recent reports have suggested that this is not always the case, with studies suggesting that some commercially available products contain significantly higher amounts of THC than stated on the label, even, in some cases, greater than the legal threshold (Gurley et al., 2020), along with other illegal cannabinoids (Poklis et al., 2019). From an athletic perspective, this is worrying not only from a legal perspective but also in terms of an anti-doping violation, given that all cannabinoids, except CBD, are currently prohibited substances by the world Anti-Doping Agency (WADA). Despite CBD being removed from the WADA prohibited list in 2018 (both in and out of competition), its use in professional sport remains highly contentious and poses a significant risk to athletes. This is not only due to the possibility of THC being present in excess of the legal threshold, but also, to date, there has been no research to suggest what effects taking varying amounts of legal CBD has on urine THC concentrations. It is for these reasons that many sporting bodies currently advise against CBD use; however, media reports would suggest that athletes are ignoring this advice and using it to enhance recovery. It is, therefore, essential that the prevalence and rationale for CBD use is studied to guide immediate education strategies and highlight areas for future research.

To this end, the aim of the present study was to establish the prevalence of CBD use in a large sample $(>500)$ of professional rugby league and union players. Rugby was chosen as the sport to study given that media reports have suggested that there are many players using CBD and because the nature of the sport is one whereby remedies to alleviate muscle soreness are frequently used. We hypothesized that CBD use (past and present) would be high $(>20 \%)$ in professional players, with an increasing prevalence in the older players and those players who play in the "forward" position.

\section{Methods}

\section{Overall Study Design}

Professional male players from 30 rugby teams competing within the English Premiership, U.K.-based Pro 14 (Rugby Union) and Super League (Rugby League) competitions were invited to participate in this observational study. Data were collected during the 2019-2020 season from 25 clubs (from the 30 invited), totaling 517 responses. At the time of data collection, all players were part of the senior squad and aged 18 years and older. All English Premiership teams and Scottish Pro 14 teams, 25\% of Welsh Pro 14 teams, and $83 \%$ of Super League teams participated (Figure 1). Players were given a short anonymous online multiple-choice survey consisting of 15 questions surrounding CBD use products, with assumed consent provided via a consent statement and submission of the survey. Each team was provided a code, only known by the lead researcher, to identify teams for relevant feedback where requested by organizations. Survey responses were coded and entered into Microsoft Excel prior to analysis. The study was approved by Liverpool John Moores University Ethics Committee (M20_SPS $966,21 / 02 / 20)$.

\section{Survey Design}

The survey was designed by the research team, with extensive experience within the elite rugby environment, to identify the current use of CBD within elite rugby and to assess athlete understanding and knowledge of key areas. A copy of the survey can be seen within Appendix. In brief, the survey was split into eight sections: (a) demographics (age, code of rugby, and positional group), (b) awareness of CBD, (c) use of CBD, (d) rationale for use, (e) experience of use, (f) efficacy of use, (g) understanding of antidoping, and (h) further comments.

\section{Data Analysis}

Data were organized based on demographic information for age group (18-23, 23-28, and $>28$ years), rugby code (league and union), player position (forwards and backs), and team. Data are presented as percentage of responses, but analysis was conducted on the frequency data. Goodness of fit chi-square $\left(\chi^{2}\right)$ was used to assess CBD use in the sample population, between codes and player position. Effect sizes were calculated using Cramer's $V$, which was interpreted as small (0.1-0.3), medium (0.3-0.5), and large (>0.5). Chi-square tests of independence were used to determine differences between age groups. Post hoc analysis was conducted by calculating Bonferroni adjusted $p$ values from adjusted standardized residuals. A Kruskal-Wallis one-way analysis of variance was used to assess differences between teams. Effect sizes were calculated using epsilon square $\left(\varepsilon^{2}\right)$ and were interpreted as weak $(<0.04)$, moderate $(0.04-0.16)$, and strong $(0.64-1.0)$, respectively. Statistical significance was set at $p<.05$, apart from Bonferroni adjusted $p$ values, which were calculated as the $p$ value from the adjusted standardized residuals divided by the degrees of freedom. All data were analyzed using SPSS for Windows (version 25; IBM, Armonk, NY).

\section{Results}

There were a total of 18 U.K.-based teams that competed within the English Premiership and Pro 14 rugby union competitions, along 


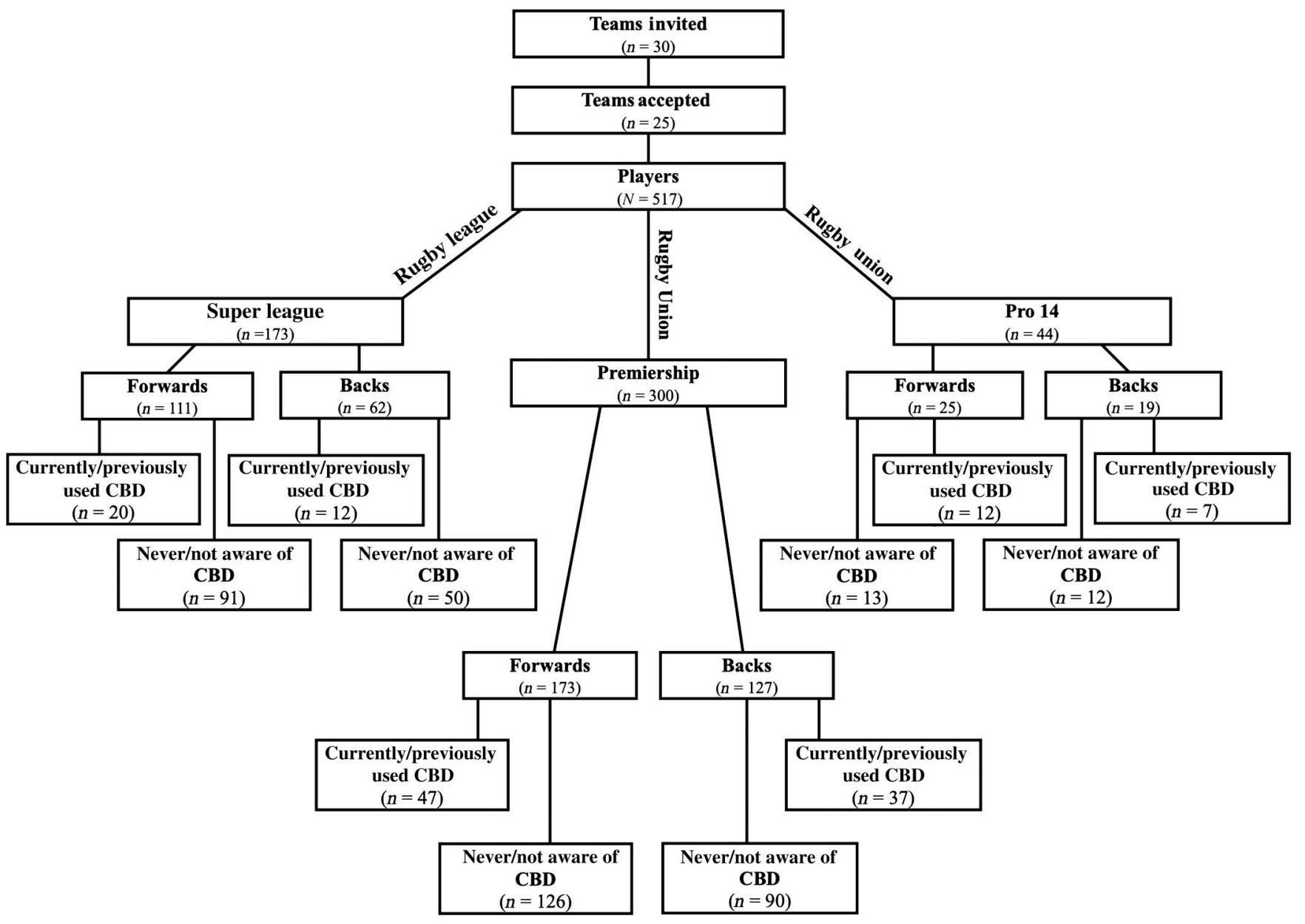

Figure 1 - Overview of recruitment for survey. $\mathrm{CBD}=$ cannabidiol.

with 12 teams that competed within the Super League rugby league competition. Out of these, 517 players from 25 teams responded to the survey (Figure 1).

\section{Demographics}

The mean age of players who responded was 25 ( $S D=5$, range 18-33) years. Sixty-seven percent (of the 517 respondents) came from rugby union, with the remaining $33 \%$ from rugby league. Of those who responded, $40 \%$ identified themselves as backs and $60 \%$ as forwards (Figure 1).

\section{Awareness and Use of CBD}

Of those responders to the survey, 91\% $(n=472)$ indicated that they had an awareness of CBD. Awareness came from a variety of sources: Internet (73\%), another teammate (61\%), another player/ club (41\%), nutritionist (16\%), other staff members (4\%), family/ friends $(2 \%)$, the media $(1 \%)$, and research $(<1 \%$; Figure $2 \mathrm{a})$. Of the 472 players that were aware of CBD (Figure 3a), significantly more players had never used it $\left(\chi^{2}=121.9, p<.001, V=0.24\right)$; however a considerable proportion (26\%) were either currently $(8 \%)$ or had previously (18\%) used CBD. The proportion of players either using or who had used CBD (Figure 4) varied significantly across the teams sampled $\left(H=278.0, p<.001, \varepsilon^{2}=0.59\right)$.
A significantly higher number of players from rugby union had either used or were using CBD $\left(\chi^{2}=8.29, p=.004, V=\right.$ $0.13)$, but there were no observed differences between backs and forwards $\left(\chi^{2}=0.09, p=.760, V=0.013\right)$. Interestingly, CBD use was significantly different across the age groups $\left(\chi^{2}=40.60\right.$, $p<.001, V=0.28)$, with a lower number of players in the 18 23 years age group $(p<.0001)$ and more than expected players using CBD in the 28 years and older age group $(p<.0001)$. This contrasted with the 23-28 years age group, which was not observed to be significantly different from the expected number of users $(p=.053)$.

\section{Rationale for Use}

Of the 472 players aware of CBD, 339 had never used CBD. The reasons surrounding this included being concerned about anti-doping rule violations (ADRV; 64\%), not aware of any benefit (37\%), not perceiving a need $(29 \%)$, club advised against taking $(23 \%)$, too costly $(2 \%)$, have not had the chance $(1 \%)$, concerned about possible side effects $(1 \%)$, and lack of scientific evidence $(<1 \%)$ (Figure $2 b)$.

For the remaining 133 players who previously/currently used $\mathrm{CBD}$, the reasons were equally varied, including improving recovery/pain (80\%), sleep (78\%), anxiety (32\%), and for other medical purposes (14\%: help with concussion, long-term injury, and chemotherapy; Figure 2c). 
(a)

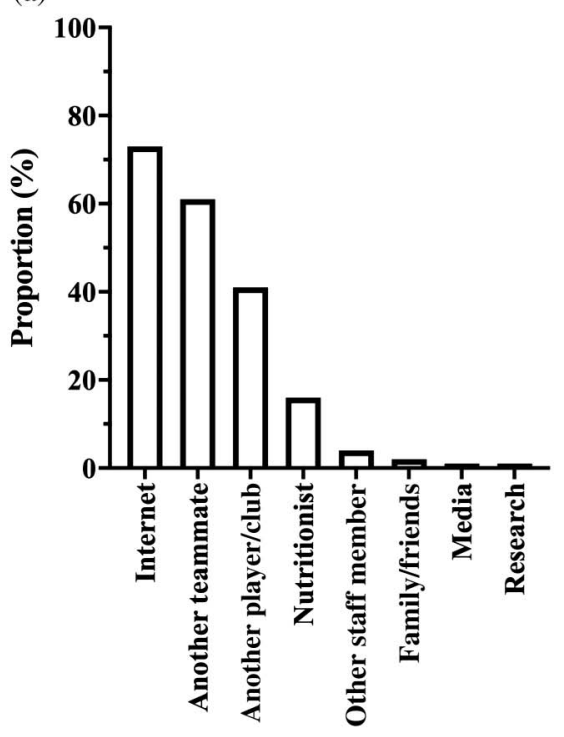

(c)

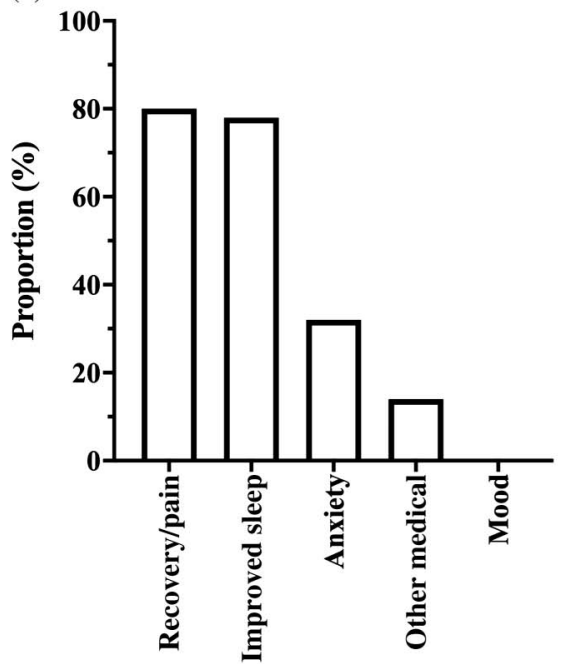

(b)

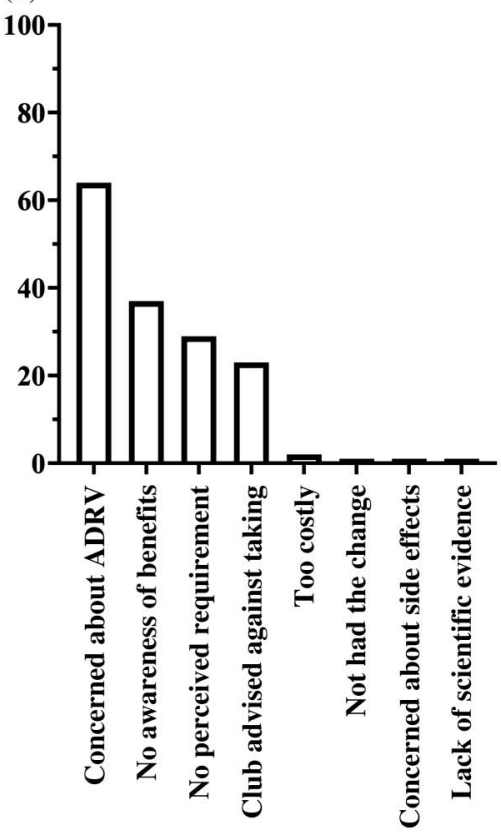

(d)

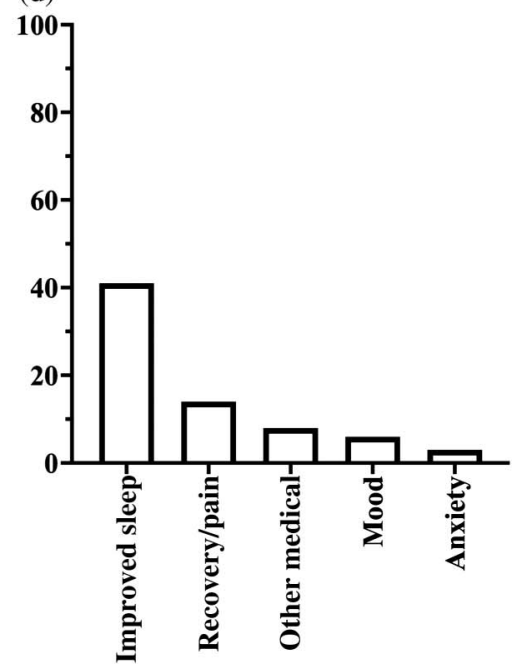

Figure 2 - Sources of information (a), reasons against (b), reasons for (c), and perceived benefits (d) of taking cannabidiol oil. ADRV = anti-doping rule violations.

\section{Experience and Efficacy of CBD Use}

Thirteen different CBD brands were being used by athletes, all of which were oils or capsules. There was a disparity in reported dose with athletes quoting percentages, drops, mgs, sprays, and "rice-sized" quantities. Indeed, there was also diversity in the concentrations of CBD reportedly taken, ranging from 400 to $3,000 \mathrm{mg} /$ $5-10 \%$ solutions in forms of drops (3-10 drops), tablets (10 mg), and "rice-sized" quantities ("one grain"). A number of players (6\%) were either unsure of the brand they were using or mixing multiple brands, with a further $39 \%$ unsure of the dose they were taking.

Despite the disparity in dose taken, many of the players currently or previously taking CBD $(n=133)$ anecdotally perceived a benefit (67\%), including improved sleep (41\%), recovery/pain $(14 \%)$, mood $(6 \%)$, anxiety $(3 \%)$, and other various medical purposes (8\%: concussion, chemotherapy, and long-term injury). No player reported any perceived adverse effects (Figure 2d).

\section{Understanding of Anti-Doping and Further Comments}

Of the players ( $n=133)$ who had taken or were currently taking CBD, $52 \%$ were not concerned of committing an ADRV, with a further $79 \%$ believing the product they use to be batch tested for banned substances and $21 \%$ unsure or aware that the product is not tested.

Of the 107 players that included additional commentary, 14\% used this space to indicate that they would try CBD in the future, with $13 \%$ still unsure of the legality in and out of sport.

\section{Discussion}

The aim of the present study was to establish the prevalence and reasons for CBD use in an extensive sample of elite rugby players. To this end, we recruited 517 professional rugby players to 

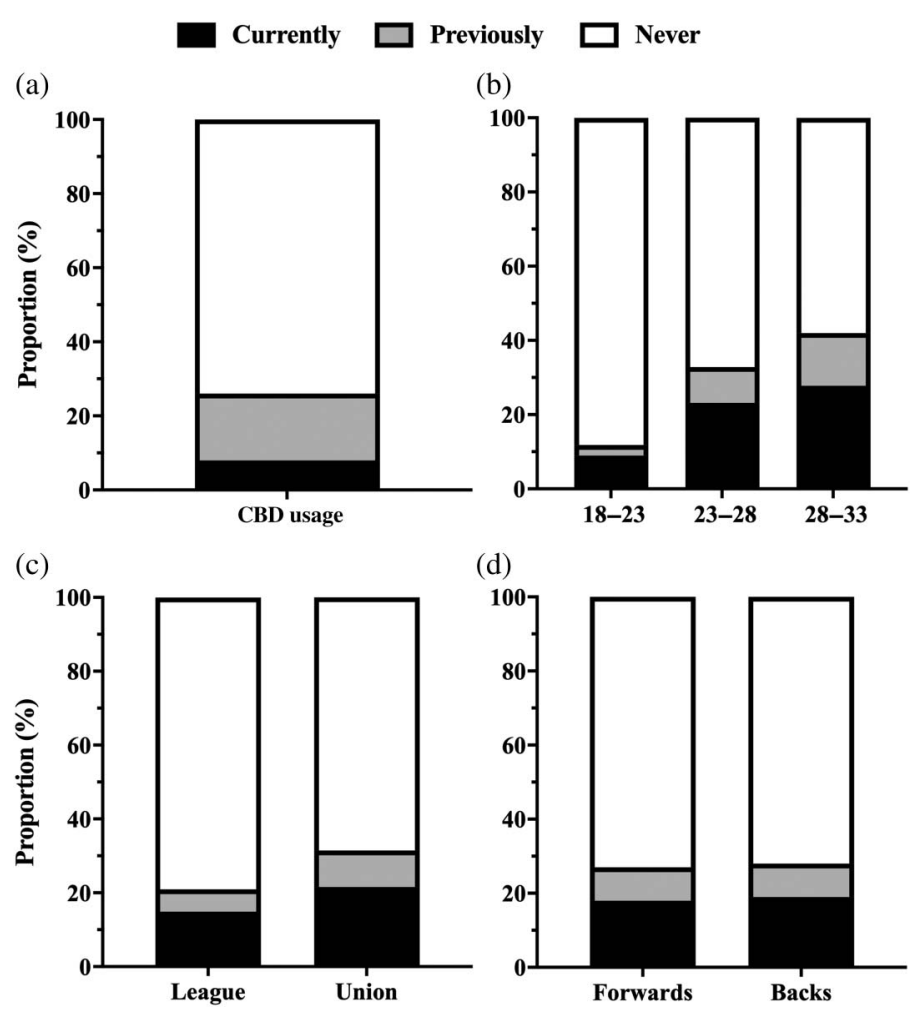

Figure 3 - Varying usage (\%) of CBD oil across (a) all surveyed players, (b) different ages, (c) different codes, and (d) different playing position. Bars represent data from those who currently (black), previously (gray), or have never (white) taken CBD oil. CBD = cannabidiol.

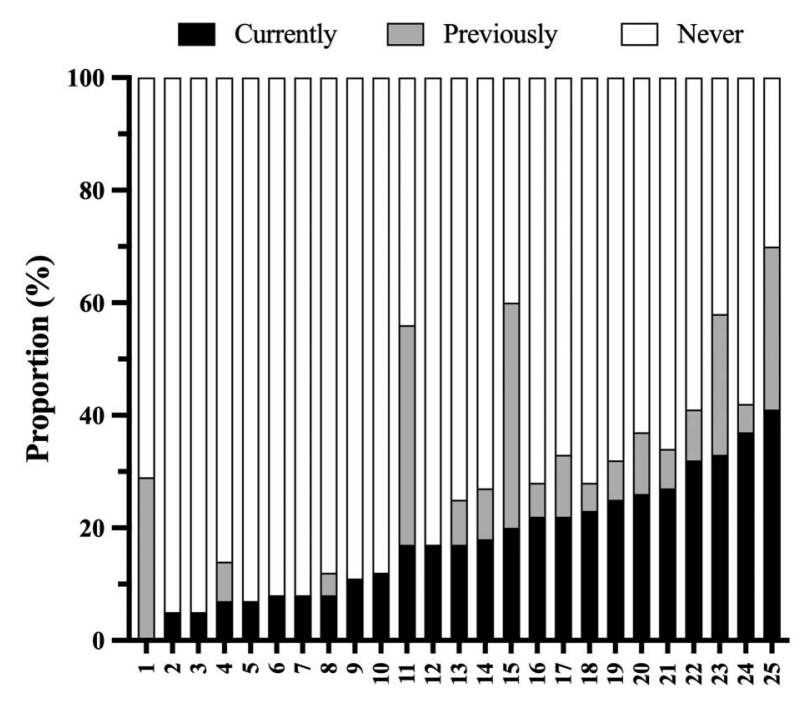

Team

Figure 4 - Individual team usage of CBD oil ranked lowest to highest for previous/current use of CBD. Bars represent data from those who currently (black), previously (gray), or have never (white) taken CBD oil. $\mathrm{CBD}=$ cannabidiol.

complete an online survey. We report, for the first time in elite rugby players, that despite warnings from clubs and national governing bodies against CBD use, $>25 \%$ of all athletes surveyed have either used or continue to use CBD. Moreover, in the older players ( $>28$ years old), almost $40 \%$ have or continue to use CBD, with the major reasons cited including pain relief/recovery and to improve sleep quality. These data suggest that many rugby players are willing to accept the risks associated with CBD use in an attempt to accelerate recovery and/or improve sleep. In addition to the definitive requirement for immediate player education with regard to the safety and risks of CBD supplementation, future research should now also assess the efficacy of CBD supplements on factors influencing recovery (e.g., pain, soreness, inflammation, sleep).

Rugby match play has been shown to result in both exerciseand impact-induced muscle damage (Twist et al., 2012) that lasts for several days postgame and persists throughout the entire playing season (Fletcher et al., 2016). Daily muscle pain, especially nerve pain, has been shown to have both physical (Cheung et al., 2003) and psychological consequences that negatively affect daily quality of life and even family life (Duenas et al., 2016). It is, therefore, of little surprise that athletes have been shown to have four times the use of painkillers compared with the general public (Alaranta et al., 2006). Although research from other sports has documented a high prevalence of painkiller use (Taioli, 2007; Tsitsimpikou et al., 2009), data on rugby players are lacking. This is despite the suggestion, both in academic literature (Gibbison et al., 2014) and multiple reports in the mainstream media, that rugby players are particularly reliant on the use of painkillers, such as non-steroidal anti-inflammatory drugs, and opiates such as Tramadol. The side effects associated with the chronic use of nonsteroidal anti-inflammatory drugs include gastric injury, gastric ulceration, and kidney damage (Bertolini et al., 2001). Importantly for rugby, increased risks also include bleeding and/or intracranial hemorrhage post minor head injury (Sakr \& Wilson, 2005). Tramadol, which has been suggested to be widely used in rugby players in the media, is effective in treating neuropathic pain (Bravo et al., 2017); however, it is also not without serious side effects, including nausea, headaches constipation, sleep disturbances (Walder et al., 2001), and withdrawal effects, as well as drowsiness, which can significantly impair training and performance. Given the muscle damage induced by rugby match play and training (Hudson et al., 2019), combined with the side effects of traditional pain medication, rugby players are now seeking alternate pain relief strategies and ways to improve sleep.

We report that $78 \%$ and $80 \%$ of players used CBD to improve sleep and pain/recovery, respectively. Interestingly, in the present study, although the mean use of CBD was $26 \%$, there was increasing prevalence of use with increasing age, with the mean usage reaching $39 \%$ in the players aged 28 years and older category. Although it was not possible to assess the reason for this, one could speculate that it is the older players who have residual pain through a longer playing history, and that they are more likely to be seeking pain relief medication. Of all the players who have used CBD, $67 \%$ reported a perceived benefit of use, with $41 \%$ reporting improved sleep and $14 \%$ improved pain/recovery. Interestingly, no athletes reported any adverse side effects, although it must be considered that players may have taken a negligible dose of CBD and/or taken a product with far less CBD than stated on the label (Gurley et al., 2020), and therefore this study should not be used as evidence that CBD does not cause side effects. Future research should now be performed to explore the veracity of such claims, which could have important consequences for future advice and guidance offered to players.

Although the mean current/previous use of CBD was $26 \%$, it was interesting to observe that there was a significant difference between teams with current/previous use ranging, from 5\% to $70 \%$. 
Alarmingly, the players reported that the major source of CBD information was either the Internet $(73 \%)$ or a teammate $(61 \%)$. It is, therefore, possible that the interteam range could be related to some players within a club having a major influence, although this suggestion could not be fully explored in the present study and should now be investigated further. This suggestion is, however, further supported by the fact that only $16 \%$ and $4 \%$ sourced their information from the team nutritionist or other staff member, respectively. This low reliance on the team nutritionist could be due to most clubs and governing bodies advising against CBD use and, as a consequence, players not feeling comfortable to discuss the potential use of CBD with internal staff members. Indeed, 23\% of players who did not use CBD cited their main reason not to use was that the club had advised against it. In the free comments section of the survey, $14 \%$ of players stated that they would like to try $\mathrm{CBD}$ in the future. It is, therefore, crucial that players feel comfortable discussing CBD with qualified staff rather than relying on the Internet and/or other team members for their information (Maughan et al., 2018).

Despite CBD being removed from the WADA prohibited list in 2018, CBD use still poses a significant risk to athletes due to the possibility of THC and other prohibited cannabinoids being present in quantities sufficient to produce a urine sample greater than the current threshold. Although $64 \%$ of players did not use CBD due to fears of an ADRV, only $48 \%$ of current/previous users were concerned about such a violation. In the free comments section, $13 \%$ of players stated that they were unsure of the legality of CBD in rugby. The survey also asked athletes if they were aware if their supplement was batch tested for other contaminants. Alarmingly, $21 \%$ of users did not know if their CBD supplement was batch tested, whereas $79 \%$ claimed that the CBD product they were consuming was tested. This belief that the product was tested was of particular concern given that of all the CBD products listed, only one appeared to be tested (tested by BSCG), yet just $39 \%$ of the players reported to be using this specific product. It is, therefore, likely that some players wrongly assumed that the CBD product they were taking was batch tested, which could be related to the lack of qualified advice they had received. Given that rates of supplement contamination, which could result in an ADRV, have been reported as high as 12-58\% (Martínez-Sanz et al., 2017), combined with the risks associated with CBD, it appears that many rugby players are prepared to risk an ADRV and subsequent sanctions in a quest to attenuate their pain and enhance recovery (including promotion of sleep). This confirms the need for appropriate education as well as greater efforts to provide appropriate pain relief for players.

The present study is not without its limitations, most of which are a consequence of collecting data in professional athletes alongside the collection of data that occurred during the COVID-19 crisis. Although we managed to recruit more than 500 players, some teams declined to participate in the research, with reasons cited including the furlough of players and the potential for confusion, given they had advised their players against using CBD. It is feasible that players who had been advised not to use CBD did not take part in the study, and, likewise, the players who did agree to participate may have had more of an interest in CBD than those who declined. This potential for systemic bias could have either overestimated or underestimated the reported prevalence, which is a common problem in survey data when the entire population is not recruited. Nonetheless, we are confident that with 25 teams and a mean of 21 players per team, these data do reflect the genuine usage within elite professional rugby. Moreover, due to the anonymous nature of the data collection, it was not possible to explore in depth the rationale for usage and the perceived benefits. It was felt that the anonymous nature was essential, given that the advice from the clubs was not to use CBD, and, by keeping it anonymous, it would allow us to collect more robust data. Future research should now consider a more qualitative approach to fully understand the decisions made by rugby players when it comes to CBD use. Finally, while players did report benefits of CBD use, this could certainly be a placebo effect, given that, to date, there are no randomized placebo-controlled trials on the effects of CBD on recovery and sleep in athletes. Future research must now address this gap in the literature as well as assess the potential for CBD use to raise THC concentrations above the current WADA thresholds.

In conclusion, we report, for the first time, a high prevalence of CBD use, along with the rationale for its use in elite rugby players. The data suggest that despite the majority of clubs advising against CBD use, many players are ignoring this advice in an attempt to improve sleep and pain management. These data highlight the need for immediate education for rugby players on the potential consequences of CBD use, as well as highlighting the need for future research into the efficacy of CBD and the veracity of the anecdotal claims from the players that it does help alleviate pain, enhance recovery, and promote sleep.

\section{Acknowledgments}

The study was designed by A.M. Kasper, M. Hooks, M. Skeer, B. Webb, H. Nia, J.P. Morton, and G.L. Close; data were collected and analyzed by A.M. Kasper, M. Hooks, S.A. Sparks, and G.L. Close; and data interpretation and manuscript preparation were undertaken by A.M. Kasper, J.P. Morton, S.A. Sparks, and G.L. Close. All authors approved the final version of the article.

\section{References}

Alaranta, A., Alaranta, H., Heliovaara, M., Airasksinen, M., \& Helenius, I. (2006). Ample use of physician-prescribed medications in Finnish athletes. International Journal of Sports Medicine, 27(11), 919-925.

Austin, D.J., \& Kelly, S.J. (2013). Positional differences in professional rugby league match play through the use of global positioning systems. Journal of Strength and Conditioning Research, 27(1), 14-19. doi:10.1519/JSC.0b013e31824e108c

Bergamaschi, M.M., Queiroz, R.H., Zuardi, A.W., \& Crippa, J.A. (2011). Safety and side effects of cannabidiol, a Cannabis sativa constituent. Current Drug Safety, 6(1), 237-249.

Bertolini, A., Ottani, A., \& Sandrini, M. (2001). Dual acting antiinflammatory drugs: A reappraisal. Pharmacology Research, 44(6), 437-450. doi:10.1006/phrs.2001.0872

Bravo, L., Mico, J.A., \& Berrocoso, E. (2017). Discovery and development of tramadol for the treatment of pain. Expert Opinion on Drug Discovery, 12(12), 1281-1291. PubMed ID: 28920461 doi:10.1080/ 17460441.2017.1377697

Cheung, K., Hume, P.A., \& Maxwell, L. (2003). Delayed onset muscle soreness. Sports Medicine, 33(2), 145-164. PubMed ID: 12617692 doi:10.2165/00007256-200333020-00005

Close, G.L., Kasper, A.M., \& Morton, J.P. (2019). From paper to podium: Quantifying the translational potential of performance nutrition research. Sports Medicine, 49(Suppl.), 25-37. doi:10.1007/s40279-018-1005-2

Cunniffe, B., Hore, A.J., Whitcombe, D.M., Jones, K.P., Davies, B., \& Baker, J.S. (2011). Immunoendocrine responses over a three week international rugby union series. Journal of Sports Medicine and Physical Fitness, 51(2), 329-338. 
Duenas, M., Ojeda, B., Salazar, A., Mico, J.A., \& Falide, I. (2016). A review of chronic pain impact on patients, their social environment and the health care system. Journal of Pain Research, 9(1), 457-467. PubMed ID: 27418853 doi:10.2147/JPR.S105892

Fletcher, B.D., Twist, C., Haigh, J.D., Brewer, C., Morton, J.P., \& Close, G.L. (2016). Season-long increases in perceived muscle soreness in professional rugby league players: Role of player position, match characteristics and playing surface. Journal of Sports Sciences, 34(11), 1067-1072. PubMed ID: 26368285 doi:10.1080/02640414. 2015.1088166

Gibbison, B., Bailey, C.R., \& Klein, A.A. (2014). Tramadol—the marmite drug. Anaesthesia, 70(2), 125-130. PubMed ID: 25491015 doi:10. 1111/anae.12972

Gurley, B.J., Murphy, T.P., Gul, W., Walker, L.A., \& ElSohly, M. (2020). Content versus label claims in cannabidiol (CBD)-containing products obtained from commercial outlets in the state of Mississippi. Journal of Dietary Supplements. Advance online publication. doi:10. 1080/19390211.2020.1766634

Hudson, J., Cole, M., Morton, J.P., Stewart, C.E., \& Close, G.L. (2019). Daily changes of resting metabolic rate in elite rugby union players. Medicine \& Science in Sports \& Exercise, 52(3), 637-644. doi:10. 1249/MSS.0000000000002169

Iffland, K., \& Grotenhermen, F. (2017). An update on safety and side effects of cannabidiol: A review of clinical data and relevant animal studies. Cannabis Cannabinoid Research, 2(1), 139-154. PubMed ID: 28861514 doi:10.1089/can.2016.0034

Martinez-Sanz, J.M., Sospedra, I., Ortiz, C.M., Baladia, E., Gil-Izquierdo, A., \& Ortiz-Moncada, R. (2017). Intended or unintended doping? A review of the presence of doping substances in dietary supplements used in sports. Nutrients, 9(10), 1093.

Maughan, R.J., Sherriffs, S.M., \& Vernec, A. (2018). Making decisions about supplement use. International Journal of Sport Nutrition and Exercise Metabolism, 28(2), 212-219. PubMed ID: 29565185 doi:10. 1123/ijsnem.2018-0009

Mechoulam, R., \& Hanus, L. (2002). Cannabidiol: An overview of some chemical and pharmacological aspects. Part I: Chemical aspects. Chemistry and Physics of Lipids, 121(1-2), 35-43. PubMed ID: 12505688 doi:10.1016/S0009-3084(02)00144-5

Naughton, M., Miller, J., \& Slater, G. (2017). Impact-induced muscle damage and contact-sport: Aetiology, effects on neuromuscular function and recovery, and the modulating effects of adaptation and recovery strategies. International Journal of Sports Physiology and Performance, 13(8), 1-24.

Pellati, F., Borgonetti, V., Brighenti, V., Biagi, M., Benvenuti, S., \& Corsi, L. (2018). Cannabis sativa $L$. and nonpsychoactive cannabinoids: Their chemistry and role against oxidative stress, inflammation, and cancer. BioMed Research International, 2018, 1691428. PubMed ID: 30627539 doi:10.1155/2018/1691428

Piomelli, D., \& Russo, E.B. (2016). The Cannabis sativa versus Cannabis indica debate: An interview with Ethan Russo MD. Cannabis Cannabinoid Research, 1(1), 44-46. PubMed ID: 28861479 doi:10.1089/ can.2015.29003.ebr
Poklis, J.L., Mulder, H.A., \& Peace, M.R. (2019). The unexpected identification of the cannabimimetic, 5F-ADB, and dextromethorphan in commercially available cannabidiol e-liquids. Forensic Science International, 294, e25-e27. PubMed ID: 30442388 doi:10.1016/j. forsciint.2018.10.019

Russo, E.B. (2019). The case for the entourage effect and conventional breeding of clinical cannabis: No "strain," no "gain." Frontiers in Plant Science, 9, 1969. PubMed ID: 30687364 doi:10.3389/fpls. 2018.01969

Russo, E.B., Guy, G.W., \& Robson, P.J. (2007). Cannabis, pain, and sleep: Lessons from therapeutic clinical trials of Sativex, a cannabis-based medicine. Chemistry \& Biodiversity, 4(8), 1729-1743. PubMed ID: 17712817 doi:10.1002/cbdv.200790150

Sakr, M., \& Wilson, L. (2005). Best evidence topic report. Aspirin and the risk of intracranial complications following head injury. Emergency Medicine Journal, 22(12), 891-892. PubMed ID: 16299207 doi: 10.1136/emj.2005.031740

Taioli, E. (2007). Use of permitted drugs in Italian professional soccer players. British Journal of Sports Medicine, 41(7), 439-441.

Tsitsimpikou, C., Jamurtas, T., Duncan-Fitch, K., Papalexis, P., \& Tsarouhas, K. (2009). Medication use by athletes during Athens 2004 Paralympic games. British Journal of Sports Medicine, 43(13), 1062-1066. PubMed ID: 19850571 doi:10.1136/bjsm.2009. 062521

Twist, C., Highton, J., Waldron, M., Edwards, E., Austin, D., \& Gabbett, T. (2014). Movement demands of elite rugby league players during Australian National Rugby League and European Super League matches. International Journal of Sports Physiology and Performance, 9(6), 925-930. PubMed ID: 24589426 doi:10.1123/ijspp.2013-0270

Twist, C., Waldron, M., Highton, J., Burt, D., \& Daniels, M. (2012). Neuromuscular, biochemical and perceptual post-match fatigue in professional rugby league forwards and backs. Journal of Sports Sciences, 30(4), 359-367. PubMed ID: 22176201 doi:10.1080/ 02640414.2011 .640707

United Kingdom Government Home Office. (2020, June). Cannabis, CBD and other cannabinoids: Drug liscencing factsheet. UK Licensing. https://www.gov.uk/government/publications/cannabis-cbd-andother-cannabinoids-drug-licensing-factsheet

van de Donk, T., Niesters, M., Kowal, M.A., Olofsen, E., Dahan, A., \& van Velzen, M. (2019). An experimental randomized study on analgesic effects of pharmaceutical-grade cannabis in chronic pain patients with fibromyalgia. Pain, 160(4), 860-869. doi:10.1097/j.pain. 0000000000001464

Walder, B., Tramer, M.R., \& Blois, R. (2001). The effects of two single doses of tramadol on sleep: A randomized, cross-over trial in healthy volunteers. European Journal of Anaesthesiology, 18(1), 3642. PubMed ID: 11270008 doi:10.1097/00003643-200101000-00006

Xiong, W., Cui, T., Cheng, K., Yang, F., Chen, S.R., Willenbring, D., . . . Zhang, L. (2012). Cannabinoids suppress inflammatory and neuropathic pain by targeting alpha3 glycine receptors. Journal of Experimental Medicine, 209(6), 1121-1134. PubMed ID: 22585736 doi: 10.1084/jem.20120242 


\section{Appendix: Cannabidiol survey}

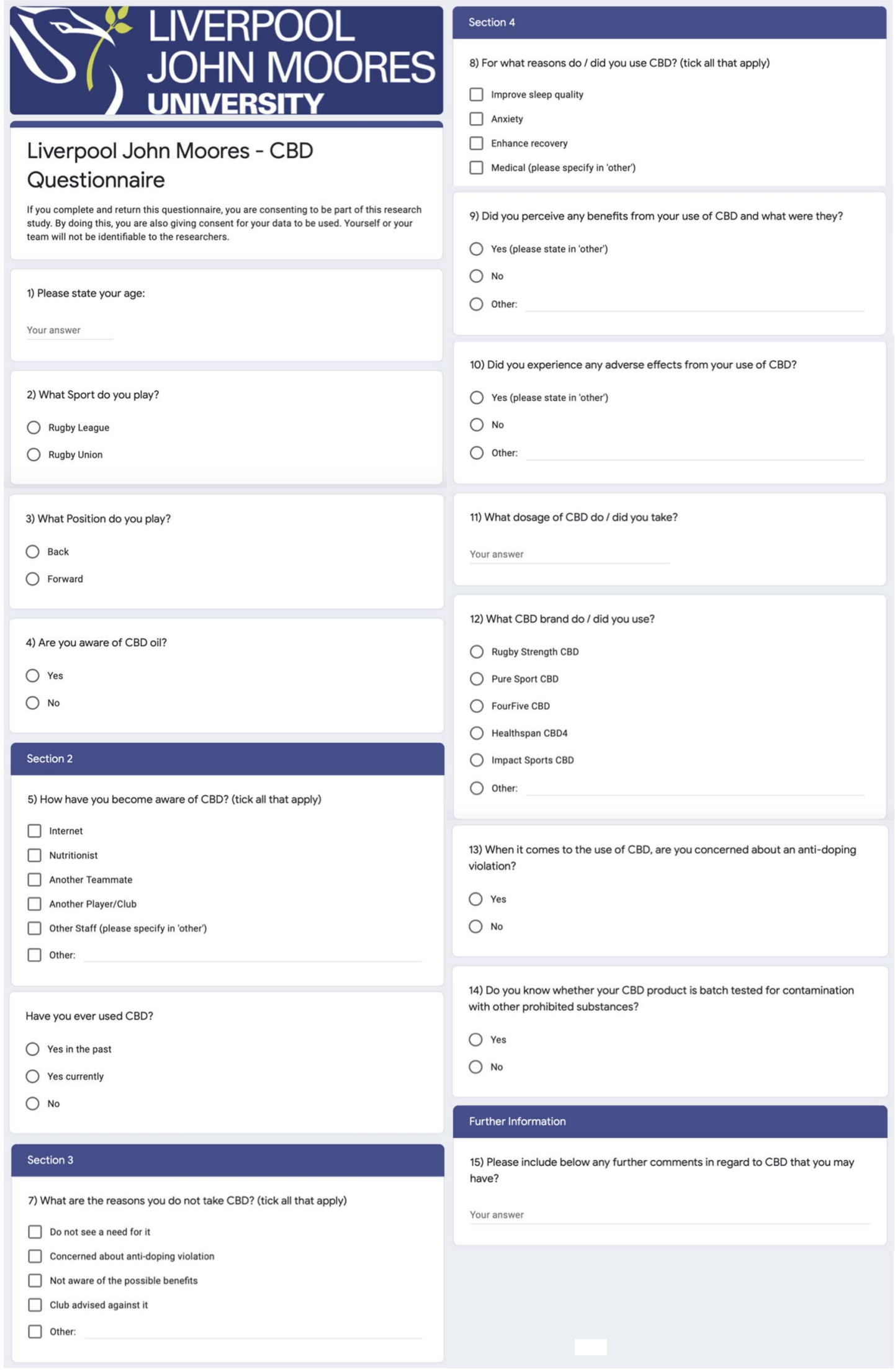

